\title{
Calculating the Ocean's Mean Dynamic Topography from a Mean Sea Surface and a Geoid
}

\author{
RORY J. BINGHAM \\ Proudman Oceanographic Laboratory, Liverpool, United Kingdom \\ Keith Haines \\ ESSC, University of Reading, Reading, United Kingdom \\ CHRis W. Hughes \\ Proudman Oceanographic Laboratory, Liverpool, United Kingdom
}

(Manuscript received 18 April 2007, in final form 12 January 2008)

\begin{abstract}
In principle the global mean geostrophic surface circulation of the ocean can be diagnosed by subtracting a geoid from a mean sea surface (MSS). However, because the resulting mean dynamic topography (MDT) is approximately two orders of magnitude smaller than either of the constituent surfaces, and because the geoid is most naturally expressed as a spectral model while the MSS is a gridded product, in practice complications arise. Two algorithms for combining MSS and satellite-derived geoid data to determine the ocean's mean dynamic topography (MDT) are considered in this paper: a pointwise approach, whereby the gridded geoid height field is subtracted from the gridded MSS; and a spectral approach, whereby the spherical harmonic coefficients of the geoid are subtracted from an equivalent set of coefficients representing the MSS, from which the gridded MDT is then obtained. The essential difference is that with the latter approach the MSS is truncated, a form of filtering, just as with the geoid. This ensures that errors of omission resulting from the truncation of the geoid, which are small in comparison to the geoid but large in comparison to the MDT, are matched, and therefore negated, by similar errors of omission in the MSS. The MDTs produced by both methods require additional filtering. However, the spectral MDT requires less filtering to remove noise, and therefore it retains more oceanographic information than its pointwise equivalent. The spectral method also results in a more realistic MDT at coastlines.
\end{abstract}

\section{Introduction}

An important challenge in oceanography is the accurate determination of the ocean's time-mean dynamic topography (MDT). If this can be achieved with sufficient accuracy for combination with the timedependent component of the dynamic topography, obtainable from altimetric data, then the resulting sum (i.e., the absolute dynamic topography) will give an accurate picture of surface geostrophic currents and ocean transports.

A geodetic estimate of the ocean's MDT can be ob-

Corresponding author address: Rory Bingham, Proudman Oceanographic Laboratory, 6 Brownlow St., Liverpool L3 5DA, United Kingdom.

E-mail: rjbi@pol.ac.uk tained by computing the difference between the mean sea surface (MSS) height obtained from satellite altimetry and the geoid height derived from a global gravity model:

$$
\eta(\theta, \phi)=H(\theta, \phi)-N(\theta, \phi),
$$

where $\theta$ is latitude and $\phi$ is longitude, and $\eta, H$, and $N$ represent the MDT, the MSS height, and the geoid height, respectively, with a reference ellipsoid providing the common datum for the MSS and the geoid. Previously this space geodetic approach has been severely limited by the accuracy of global geoid models (Wunsch and Gaposchkin 1980; Stammer and Wunsch 1994; Tapley et al. 1994; Losch and Schröter 2004). Recently, however, the Gravity Recovery and Climate Experiment (GRACE) mission has gone some way to overcoming this limitation by producing geoid models 
that represent an order of magnitude improvement in accuracy over previous satellite-based models (Tapley et al. 2003, 2005), while the forthcoming Gravity Field and Steady-State Ocean Circulation Explorer (GOCE) mission promises to go even further with a target accuracy of $1 \mathrm{~cm}$ down to spatial scales of $100 \mathrm{~km}$ (LeGrand 2001).

The most detailed picture of the ocean's mean surface circulation is obtained by combining the geodetic estimation of the MDT with finer scale in situ observations. And, in spite of the aforementioned improvements in geoid determination, this will most likely remain the case. A number of examples of this approach have been presented (e.g., Rio and Hernandez 2004; Maximenko and Niiler 2005). Nevertheless, it remains a worthwhile objective to maximize the oceanographic information that can be gleaned by purely geodetic means. To do this requires careful consideration of how best to combine the MSS and geoid surfaces. Such is our intention with this paper.

Although the geodetic MDT calculation is conceptually simple, complications arise for the following reasons. (i) The spatial variations of $H$ and $N$ are approximately two orders of magnitude larger than the spatial variations in $\eta, O(1 \mathrm{~m})$. Thus, we are looking for the small residual MDT from the difference of two larger fields, and therefore the MDT computation is extremely sensitive to any error in either the geoid or MSS-even a $1 \%$ error in either field can lead to errors in the MDT that are of the same order of magnitude as the MDT itself. (ii) A solution for the earth's gravity field is most naturally expressed, and usually supplied, as a spectral model, consisting of a set of spherical harmonic coefficients. On the other hand, the MSS, which of course is defined only over the ocean, is available to oceanographers as a gridded product. These are clearly incompatible, and must therefore be made consistent before the required difference can be obtained.

Given that the MDT is required by oceanographers in geographical form, the obvious and most straightforward approach is to express the geoid height geographically so that it can then be subtracted in a pointwise fashion from the MSS, as in Eq. (1). We refer to this as the pointwise approach. Jayne (2006) provides an example of this approach using a GRACE geoid model. The critical step in estimating the pointwise MDT is the computation of the gridded geoid height field. This is defined as the height of the geoid relative to a reference ellipsoid, which, for the purpose of calculating the MDT, should be that to which the MSS is referred. Once the geoid height has been computed it is a simple matter to subtract it from the gridded MSS to yield the

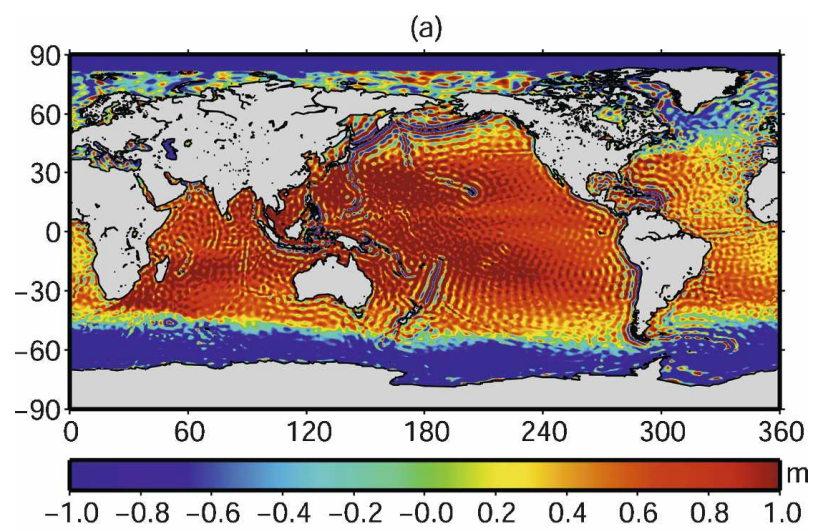

(b)

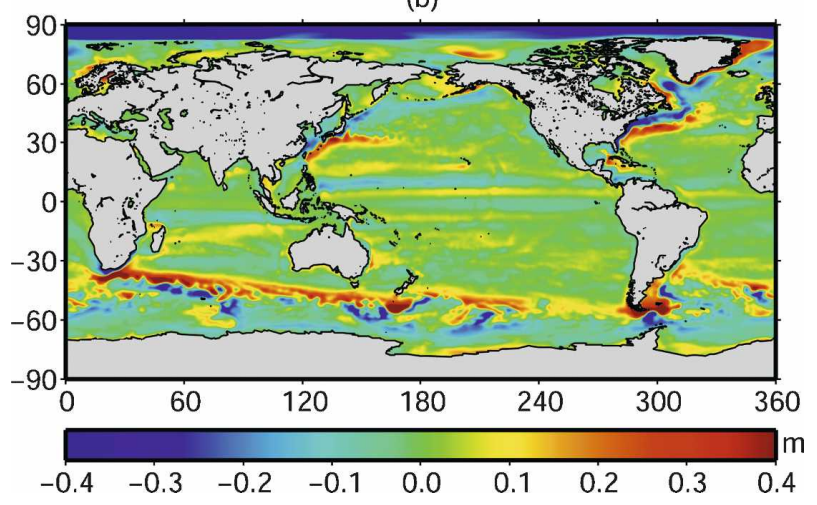

FIG. 1. (a) An MDT computed in a pointwise fashion with the geoid model (GGM02S) truncated at degree 100. (b) The component of the OCCAM MDT removed by the application of a Gaussian filter of $400-\mathrm{km}$ half-weight radius.

ocean's MDT. A thorough discussion of the subtleties of geopotential modeling is provided by Smith (1998), and full details of the geoid height computation as it pertains to oceanography can be found in Hughes and Bingham (2008).

In computing the MDT one has to choose a degree $L$ at which to truncate the expansion of geoid, limited by the maximum degree of the supplied model. Since degree $L$ corresponds approximately to a spatial scale, or half wavelength, of $20000 \mathrm{~L} \mathrm{~km}^{-1}$ the gridded geoid height field will not include spatial scales less than this. A pointwise MDT computed using a geoid truncated at degree 100 is shown in Fig. 1a. We see that while the gross features of the ocean's gyre circulation can be discerned, the detail is obscured by noise. The primary source of this noise is geoid omission error due to the truncation of the spectral geoid model.

Omission errors in a spectral model manifest themselves in two ways when projected into the spatial domain: one local, corresponding to the absence of smallscale physical features in the true field, and the other nonlocal, corresponding to Gibbs oscillations, which decay slowly away from discontinuities or sharp gradi- 
ents in the field. Both these aspects of the geoid omission error, although small in comparison to the magnitude of the geoid itself, if not mitigated, lead to large MDT errors, the magnitude of which depend inversely on the truncation degree $L$. A further source of error, which, unlike omission error, increases exponentially with $L$, is the geoid commission error. However, as shall be shown below, for $L<100$ omission error is the dominant source of error in the MDT.

With the pointwise approach local errors of omission in the gridded geoid leak into the MDT because the MSS contains small scales missing from the geoid. This means that upon differencing the MDT retains these geodetic features as errors, most prominently at small scale but large-amplitude features of the ocean floor topography, such as ridges, trenches, fracture zones, and seamounts (Tapley et al. 2003). It is in the vicinity of these features that nonlocal geoid omission error also has its greatest impact on the pointwise MDT. But the nonlocal nature of the Gibbs oscillations means that even in regions, such as the abyssal plains, where there are few small-scale geodetic features to resolve, geoid omission error leads to significant contamination of the MDT. Moreover, even steep gradients in the geoid over land can lead to large errors in the computed MDT. This is apparent, for instance, in the southeast Pacific along the Andean edge of South America.

The nonlocal nature of Gibbs oscillations, which result in substantial topography over land leading to errors over the ocean, has previously been identified in the context of spectral climate models (e.g., Hoskins 1980; Lindberg and Broccoli 1996).

Note that, while the application of a spectral domain smoothing kernel, such as that proposed by Jekeli (1981), can reduce the nonlocal Gibbs omission error in the gridded geoid, any improvement in the MDT is more than offset by a concomitant increase in MDT errors from local geoid omission error.

The common solution to the problem of geoid omission error contamination of the MDT is to use a spatial averaging filter to smooth the MDT. Many filter kernels can be defined, each with its own spectral characteristics. Jayne (2006) for example uses a Hamming window. Yet, whichever filter is chosen, there are a number of problems with spatial smoothing. First, there is the issue of signal attenuation, whereby, in addition to removing unwanted noise, meaningful oceanographic information is also lost. This is illustrated in Fig. $1 \mathrm{~b}$ where we have subtracted from an MDT generated by a high-resolution global ocean data assimilation experiment (Fox and Haines 2003) using the 1/4 $4^{\circ} 36$-level Ocean Circulation and Climate Advanced Modelling Projects (OCCAM) model (Webb et al. 1997) the same
MDT smoothed with a 400-km Gaussian filter. The OCCAM MDT is obviously not contaminated by geoid errors and therefore the undesired consequence of filtering manifests itself clearly as the parallel positive and negative bands that appear in regions of strong ocean currents such as the Antarctic Circumpolar Current (ACC), the Gulf Stream, the Kuroshio Current and its extension, and the equatorial currents. The effect of filtering is to "smear out" the MDT gradient, resulting in weaker and less well-defined estimates of the ocean currents. Because the degree of signal attenuation is proportional to the width of the filter used, it is important to find the minimum filter radius sufficient to remove the noise. A further issue is how to best to apply the filter where it encompasses grid points where the MDT is undefined, such as over land. Setting such grid points to zero in the filtered value causes attenuation in coastal regions, whereas excluding them modifies the spectral characteristics of the filter.

The issue of signal attenuation in particular motivates the consideration of alternatives to the pointwise approach that may reduce the need for spatial smoothing, thereby allowing us to obtain the maximum oceanographic information from the data. Tapley et al. (2003) use a spectral approach whereby the MSS is first expressed in terms of spherical harmonic coefficients. Truncating the spectral model of the MSS then ensures that both the MSS and the geoid represent the same spatial scales. The computation of spherical harmonics requires a globally defined surface, and Tapley et al. (2003) use a geoid based on Earth Gravity Model 1996 (EGM96) to form a globally defined MSS-geoid hybrid surface. However, by distinguishing between the local and nonlocal effects of omission error we find that forming a hybrid surface using the same geoid as that used in the actual MDT calculation gives better results. In this study we also assess the relative merits of the pointwise and spectral approaches. As shown in detail below the benefit of the spectral method is that it minimizes the negative effects of spatial smoothing and difficulties encountered in coastal regions. Moreover, it allows us to distinguish more readily between the impact of the geoid omission and commission errors on the MDT, which in turn allows us to determine a limit to the spatial scales that can be resolved with the data.

The remainder of the paper is structured as follows. In the next section the gravity and altimetry datasets used in the study are described. In section 3 we present the spectral MDT method and describe how the problems associated with the MSS not being defined globally may be overcome. In section 4 we assess the spectral method against the pointwise approach and demonstrate the superiority of the former. In section 5 we 


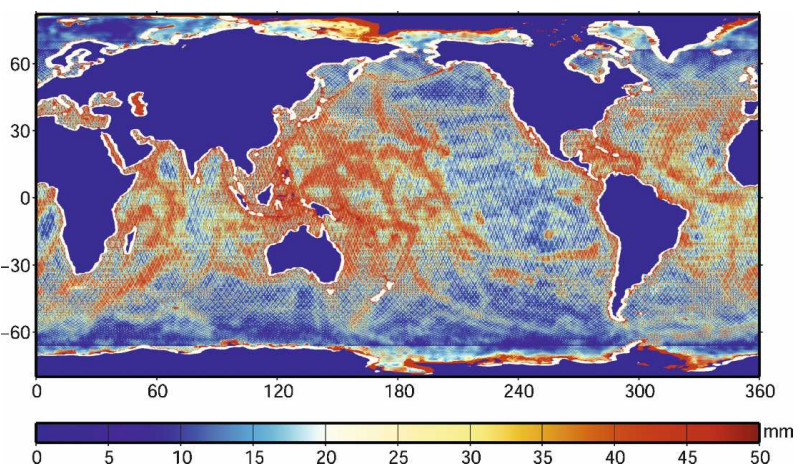

FIG. 2. The error field for the CLS01 mean sea surface. The dark blue denotes regions where the MSS is undefined and EGM96 geoid heights are used instead. The white areas adjacent to coastlines indicate where interpolation has been used to join the true MSS to the geoid. Note that this results in many islands consisting entirely of interpolated values.

draw conclusions from the work presented regarding the estimation of the ocean's MDT using geodetic data.

\section{Data}

\section{a. Altimetry data}

The most recent MSS calculated by Collecte Localisation Satellites (CLS), designated MSSCLS01 (but here shortened to CLS01), provides the altimetric dataset used in this study. This surface corresponds to the 1993-99 time mean period and is derived from $7 \mathrm{yr}$ of Ocean Topography Experiment (TOPEX)/Poseidon data, $5 \mathrm{yr}$ of European Remote Sensing Satellite (ERS1/2) repeat-track and geodetic data, and $2 \mathrm{yr}$ of Geosat data, and is defined globally between $80^{\circ} \mathrm{S}$ and $82^{\circ} \mathrm{N}$ (these limits being set by the orbital configuration of the satellites). CLS01 is defined on a regular grid with a high spatial resolution of 2 arc minutes, based on the nonrepeating geodetic sampling of the ERS and Geosat missions. Along the repeated altimeter tracks the sea surface height ( $\mathrm{SSH})$ is well sampled and the errors in CLS01 are small (see Fig. 2). In between these tracks the sea surface height is only sampled once or twice during the geodetic mission phases and thus the errors are larger. The largest errors, rising to $4 \mathrm{~cm}$, can be found in the tropics where SSH variability is high, and where the satellite ground tracks are farthest apart. (For further details see Hernandez and Schaeffer 2001.)

\section{b. Gravity model}

Currently, the best global gravity model for oceanographic purposes is undoubtedly provided by the GRACE satellite mission. The second GRACE gravity model, designated GGM02, was based on 363 days of data spanning the period from April 2002 to December 2003 (Tapley et al. 2005). Two variants are provided by the Center for Space Research (CSR) in Texas, the first-GGM02S-uses GRACE gravity data only, and is supplied as a set of spherical harmonic coefficients complete to degree and order 160 . This corresponds to a spatial resolution of approximately $125 \mathrm{~km}$. The second model-GGM02C - is constrained by additional terrestrial gravity and MSS information, and is supplied to degree and order 200. However, due to the lack of transparency in the contribution made to the geoid from these additional data sources, in this analysis we focus on the satellite-only solution (except in the forming a hybrid MSS-geoid surface for the spectral method, where we use GGM02C).

Presently, an error field is not supplied with the GRACE gravity models. However, results published by Tapley et al. (2005, see their Fig. 4) show that for GGM02S expanded to degree 70 the global RMS error is approximately $7 \mathrm{~mm}$, with a maximum of approximately $9 \mathrm{~mm}$ at the equator where the ground track spacing of the satellites is greatest. The error field is also zonally homogeneous, with no differences apparent between land and sea.

\section{c. Grid manipulations}

With the pointwise approach, the geoid height field is computed on a global grid of $0.5^{\circ}$ resolution in both longitude and latitude, and bicubic spline interpolation is used to define the MSS on the same grid. The spectral method involves replacing appropriate values in the supplied CLS01 surface with GGM02C values. To do this GGM02C geoid heights are computed on a $0.5^{\circ}$ global grid. Bicubic interpolation is then used to project the geoid height field onto the CLS01 grid, and the appropriate values in the supplied CLS01 surface, identified by flags in the accompanying error field, are replaced. Bicubic interpolation is then used again to return the hybrid surface to the $0.5^{\circ}$ global grid from which the spectral model is computed. Ideally, we would avoid interpolation by computing the geoid height field on the CLS01 grid directly from the spherical harmonic coefficients, and computing the spectral model from the high-resolution hybrid surface. However, this is rather computationally demanding, and in any case errors introduced by the required interpolations are small.

\section{The spectral method}

To truncate a spectral geoid model to obtain a gridded geoid height field is to apply a low-pass boxcar filter in the spectral domain. This filter acts to smooth 
the field, and is an ideal filter in the sense of having a perfectly defined cutoff frequency. Such a filter admits (stops) all frequencies below (above) the cutoff frequency, regardless of their amplitude. However, such ideal filters are problematic in practice because they lead to Gibbs oscillations if the field includes discontinuities or even just steep gradients. To ensure the MSS includes only those scales represented by the filtered geoid height field, it too must be filtered, otherwise the MDT will include small-scale geodetic features that are present in the MSS but not in the truncated geoid height field. The Gibbs oscillations in the geoid height field must also be removed to prevent them contaminating the MDT. The standard approach is to filter the MDT (equivalent to filtering the geoid and MSS separately) with a spatial averaging filter to remove both of these effects. However, this type of filter lacks the ideal characteristics of truncation in the spectral domain, meaning some fraction of the power at frequencies within the transition zone is admitted. Therefore, in effectively removing the noise we also attenuate scales in the MDT that ideally should be retained. [See Emery and Thomson (2001) for a more details of the issues of Gibbs oscillations and ideal filters.]

Suppose the spatial equivalent of the spectral boxcar filter can be obtained. Applying this filter to the MSS would then ensure that both the MSS and the geoid height field represented the same physical scales. Also, the attenuation of the scales to be retained, which occurs with spatial averaging filters, would be avoided. Moreover, since the MSS is very similar to geoid, filtering the MSS would lead to similar Gibbs oscillations in the vicinity of any steep gradients in the gravity field over the ocean as occur in the truncated geoid. Upon subtracting to obtain the MDT these would negate each other leaving the MDT free from Gibbs oscillations due to significant seafloor topography. However, many of the Gibbs oscillations in the gridded geoid height field over the ocean are due to continental topographic features. This problem can be resolved by creating a hybrid globally defined surface that uses geoid height as a proxy for the MSS over land. When filtered, this hybrid surface would include all of the Gibbs oscillations that are present in the gridded geoid height field, resulting in an MDT free from Gibbs oscillations.

While the above spatial filtering approach is theoretically possible, in practice a much more straightforward and computationally efficient method is to compute a set of spherical harmonic coefficients for the MSS/ geoid hybrid surface. This spectral model of the hybrid surface can be truncated in exactly the same way as the geoid is truncated, leading to similar omission errors, local and nonlocal, in both surfaces. In fact, the differ- ence between the MSS and the geoid can be taken in the spectral domain before expressing the MDT geographically. The remainder of this section is devoted to describing this method in detail.

\section{a. Mathematical background}

Suppose for the moment that the ocean covered the earth's entire surface so that the MSS was a globally defined field. Then, from Eqs. (1) and Bruns formula [Eq. (23) of Hughes and Bingham (2008); see also Smith (1998) for a discussion], and given the orthogonality of the spherical harmonic functions, it is straightforward to show that the equivalent spherical harmonic coefficients $C_{l, m}^{H}$ and $S_{l, m}^{H}$, where $l$ is the degree and $m$ the order, for the disturbing potential that would exist if the geoid was identical to the $\operatorname{MSS}(N+\eta)$, would be given by the global integral:

$$
\begin{aligned}
\left\{\begin{array}{c}
C_{l, m}^{H} \\
S_{l, m}^{H}
\end{array}\right\}= & \frac{r_{e}^{l+1}}{4 \pi G M a^{l}} \int_{S} \gamma\left(\theta^{\prime}\right) H(\theta, \phi) \\
& \times\left\{\begin{array}{c}
\cos (m \phi) \\
\sin (m \phi)
\end{array}\right\} \tilde{P}_{l, m}(\cos \theta) d s,
\end{aligned}
$$

where $\tilde{P}_{l, m}$ are the associated Legendre polynomials, $r_{e}$ is the radial distance to the surface of the reference ellipsoid, $a$ is the earth's semimajor radius, $G M$ is the earth's gravitational mass constant, and where $\gamma$ is the normal gravity on the surface of the reference ellipsoid, given by the formula of Somigliana [see Moritz (1980), his Eq. (2.16)], where $\theta^{\prime}$ is geodetic colatitude. The spectral representation of the global MDT would then be given by

$$
(C, S)_{l, m}^{\eta}=(C, S)_{l, m}^{H}-(C, S)_{l, m}^{N} .
$$

This subtraction would be possible up to the maximum degree $L$ provided by the geoid model, and the MDT could then be expressed spatially using

$$
\begin{aligned}
\eta(\theta, \phi)= & \frac{G M}{r_{e} \gamma\left(\theta^{\prime}\right)} \sum_{l=2}^{L}\left(\frac{a}{r_{e}}\right)^{l} \sum_{m=0}^{l} \tilde{P}_{l, m}(\cos \theta)\left[C_{l, m}^{\eta} \cos (m \phi)\right. \\
& \left.+S_{l, m}^{\eta} \sin (m \phi)\right],
\end{aligned}
$$

again limited by the maximum degree $L$ of the coefficients available.

However, in reality, of course, the MSS is not a globally defined field. This leads to the problem of what value to assign undefined regions in the global integration of Eq. (2). If one were to use Eq. (2) to calculate a set of spherical harmonic coefficients for the MSS using 0 (or some other constant) for undefined regions, then its expansion back into geographical space would be 
(a)

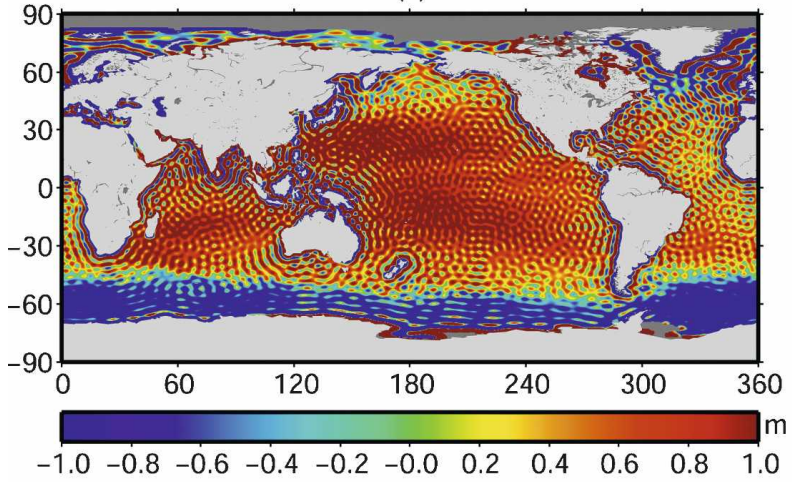

(b)

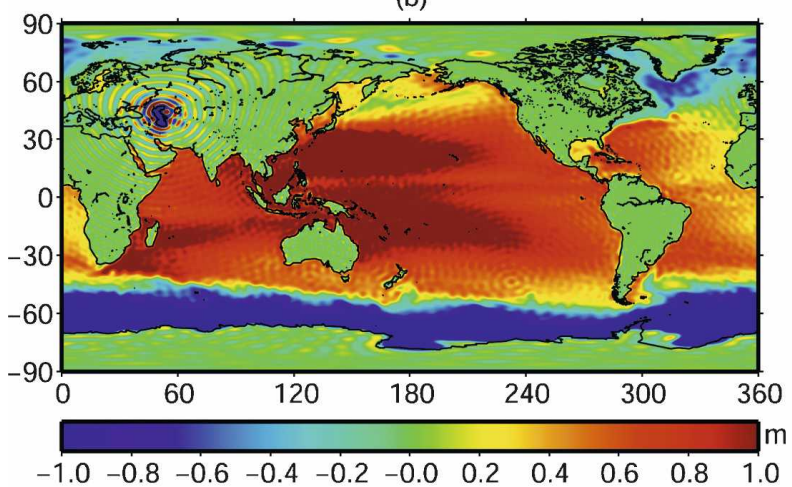

(c)

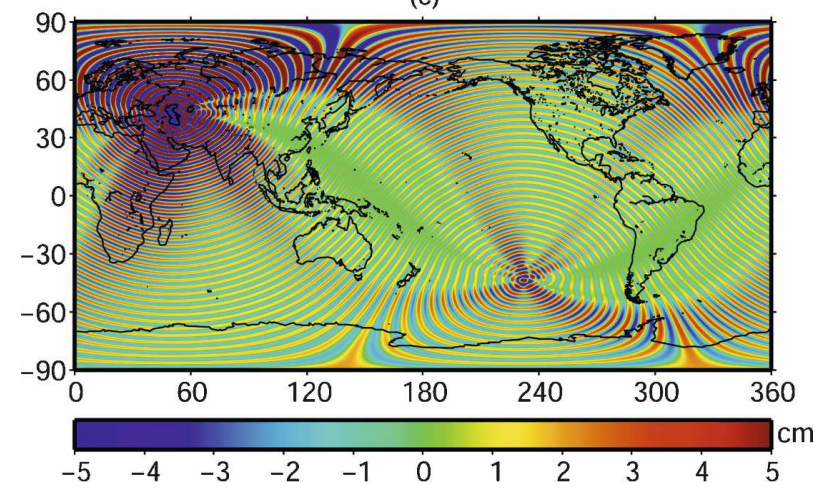

FIG. 3. (a) An MDT computed by the spectral method with grid points not corresponding to true MSS values set to 0. (b) As in (a), but with grid points not corresponding to true MSS values set equal to the geoid model GGM02C. (c) The difference between the MDT shown in (b) and a similar MDT but with the Caspian Sea in the MSS also replaced by GGM02C. All fields are expanded to degree and order 100.

severely contaminated by Gibbs oscillations due solely to the discontinuities between defined and undefined regions. These Gibbs oscillations would then contaminate the MDT, especially near coastlines where the amplitude of the Gibbs oscillations would be at their greatest (see Fig. 3a). Since many important currents occur in precisely these boundary regions this is highly unde- sirable. We now consider how best to deal with this problem.

\section{b. Producing a globally defined surface}

Since the MSS is to a close approximation equal to the geoid, the problems caused by undefined regions can be greatly reduced by using a geoid as a proxy to produce a globally complete MSS/geoid hybrid surface. Here we use GGM02C expanded to degree and order 200 as the proxy in order to get as close as possible to the observed gravity field. (However, this makes little difference compared with using GGM02S expanded to degree and order 160 , with some smoothing to suppress the commission error.) The improvement that using a proxy yields is illustrated by Fig. $3 b$, which shows the MDT produced using the hybrid surface. Now all the significant features of the ocean's gyre circulation are clearly resolved. Yet the MDT still retains significant Gibbs oscillations. It is clear from Fig. $3 b$ that these oscillations radiate from the Caspian Sea, and Fig. 3c reveals that, overlooked, they represent significant global contamination of the resulting MDT; a good example of the nonlocal impact of the Gibbs oscillations. Inspection reveals that they arise because the Caspian MSS, at least as it is defined by the CLS01 product, lies several tens of meters below the geoid. The solution is to replace the Caspian MSS in the hybrid surface with the geoid. We found that the Caspian Sea is the only inland sea that gives rise to significant Gibbs oscillations.

In fact, the CLS01 MSS is supplied as a continuous hybrid surface that is defined globally between latitudes $80^{\circ} \mathrm{S}$ and $82^{\circ} \mathrm{N}$ using the geoid model EGM96 as a proxy where the MSS is undefined (shown in dark blue in Fig. 2). In coastal regions interpolation has been used to smoothly join the MSS to the geoid, and these regions are shown with white borders around the landmasses in Fig. 2. However, the best possible matching of Gibbs oscillations in the MSS/geoid hybrid surface and the geoid used to compute the MDT, and therefore the smallest Gibbs residual in the MDT, is obtained when the difference between the geoid used in the hybrid surface and the geoid subtracted to calculate the MDT is small. As Fig. 4a shows, over land and in other undefined regions there are large differences between the EGM96 geoid heights used in CLS01 surface and geoid heights from GGM02S. These differences results in Gibbs oscillations in the regridded supplied CLS01 surface over the ocean, which are substantially different to the Gibbs oscillations in the gridded GGM02S geoid height field. This results in residual Gibbs oscillations in the MDT (see Fig. 4b). This problem is particularly acute in the Southern Ocean, southeastern Pacific, and 
(a)

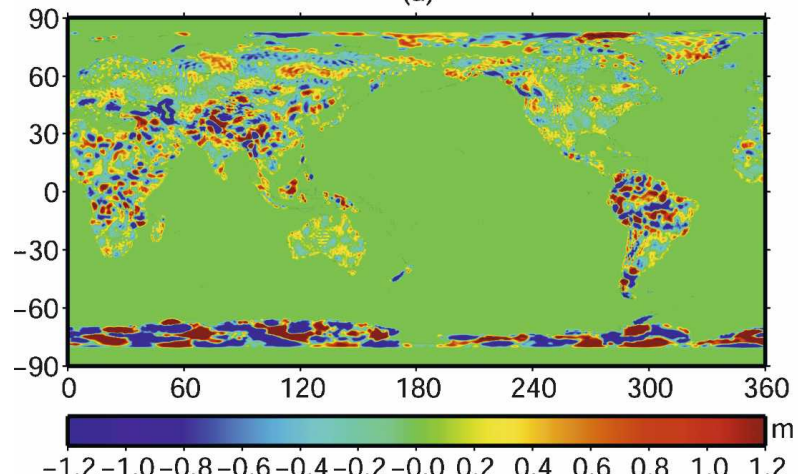

(b)

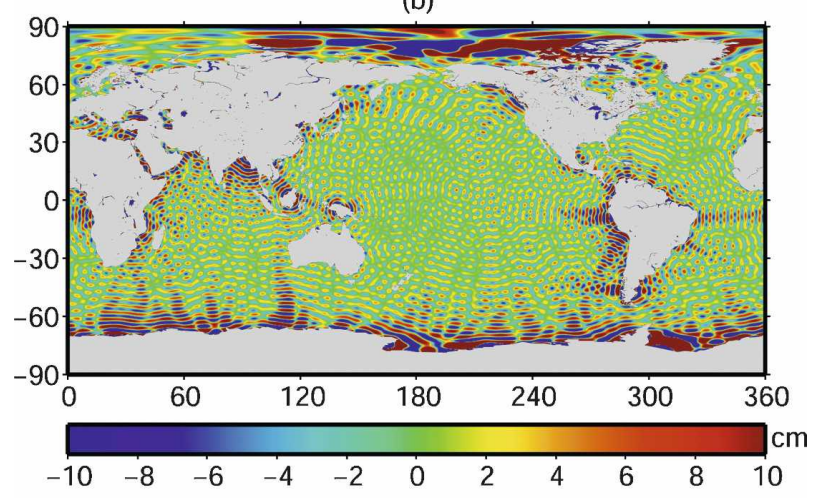

(c)

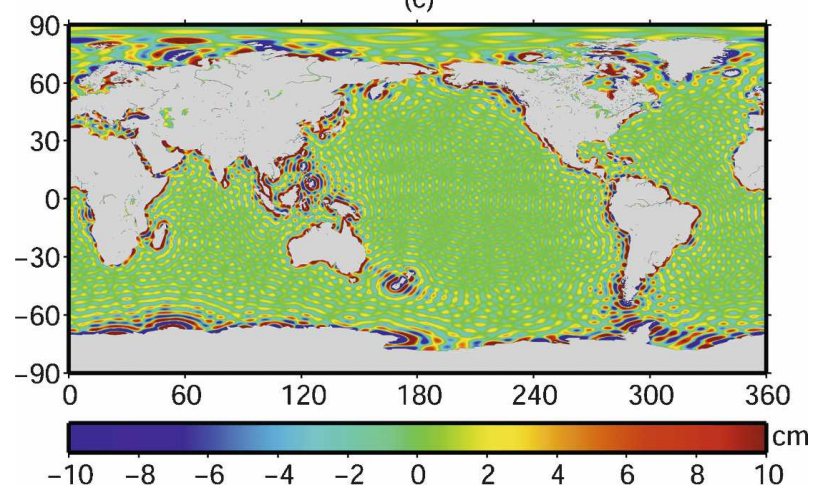

FIG. 4. (a) The difference between EGM96 and GGM02S over undefined MSS regions. (b) The difference between a spectral MDT computed to degree 100 using the CLS01 MSS as supplied with EGM96 in undefined regions and a spectral MDT with GGM02C replacing EGM96. (c) As in (b), but with GGM02C replacing EGM96 and interpolated regions. All fields are expanded to degree and order 100.

the South Atlantic, in the vicinity of the greatest differences in the GGM02S and EGM96 geoids.

The supplied CLS01 MSS smoothly joins, by interpolation, the true MSS to the EGM96 geoid. This contrasts with our hybrid surface, which includes a discontinuity, equal in magnitude to the MDT, where the MSS meets the geoid. This discontinuity will also lead to Gibbs oscillations in the computed MDT. Therefore, we considered keeping the interpolation region by only replacing actual EGM96 values with GGM02C values in an attempt to reduce this discontinuity. However, the difference between the Gibbs oscillations due to gradients in the interpolated zones-a combination of the effect of interpolation and the actual differences between EGM96 and GGM02C in these regions-leads to significant Gibbs oscillations remaining in the computed MDT (see Fig. 4c). The magnitude of these are greater than those that result from the discontinuity between the MSS and GGM02C. Therefore, both EGM96 and interpolated regions in the supplied CLS01 MSS are replaced with GGM02C.

Incidentally, the difference between the original CLS01 surface expressed as a set of spherical harmonic coefficients and then regridded and the same field but with the Caspian Sea region replaced with GGM02C is nearly identical to the difference shown in Fig. 3c. Because, for the original surface, the true MSS is smoothly joined to EGM96, resulting in a steep, but finite, sided depression in the Caspian Sea region, this confirms that it is not only true discontinuities, but also steep gradients, that give rise to Gibbs oscillations. (See also Hughes and Bingham 2008.)

\section{Comparison of methods}

We start by comparing unfiltered pointwise MDTs with spectral MDTs along a zonal section crossing the Atlantic at $30^{\circ} \mathrm{N}$ (Fig. 5). The objective of this comparison is not to establish the superiority of the spectral approach. This will require a comparison of filtered MDTs. Rather, it serves to illustrate how geoid errors in the MDT depend on the truncation degree, the problems eliminated by the spectral method, and the relative contributions made by the geoid commission and omission errors to the MDT error budget. This, in turn, informs our choice of truncation degree and smoothing filter radius (since, as we shall see, as well as filtering the pointwise MDT, it is still necessary to spatially filter the spectral MDT). To guide our expectations of the magnitude and spatial scales to be seen in the profile, each geodetic MDT profile is superimposed on the MDT profile derived from the OCCAM ocean assimilation experiment. The main features of the subtropical North Atlantic circulation can be seen in the OCCAM MDT section. The strong positive gradient in the west corresponds to the Gulf Stream, while the more gentle negative gradient toward the east corresponds to the weaker interior equatorward flow.

Figure 5 shows that the contamination of the pointwise MDT due to geoid omission error is greater than magnitude the MDT itself for all values of $L$. Clearly it 

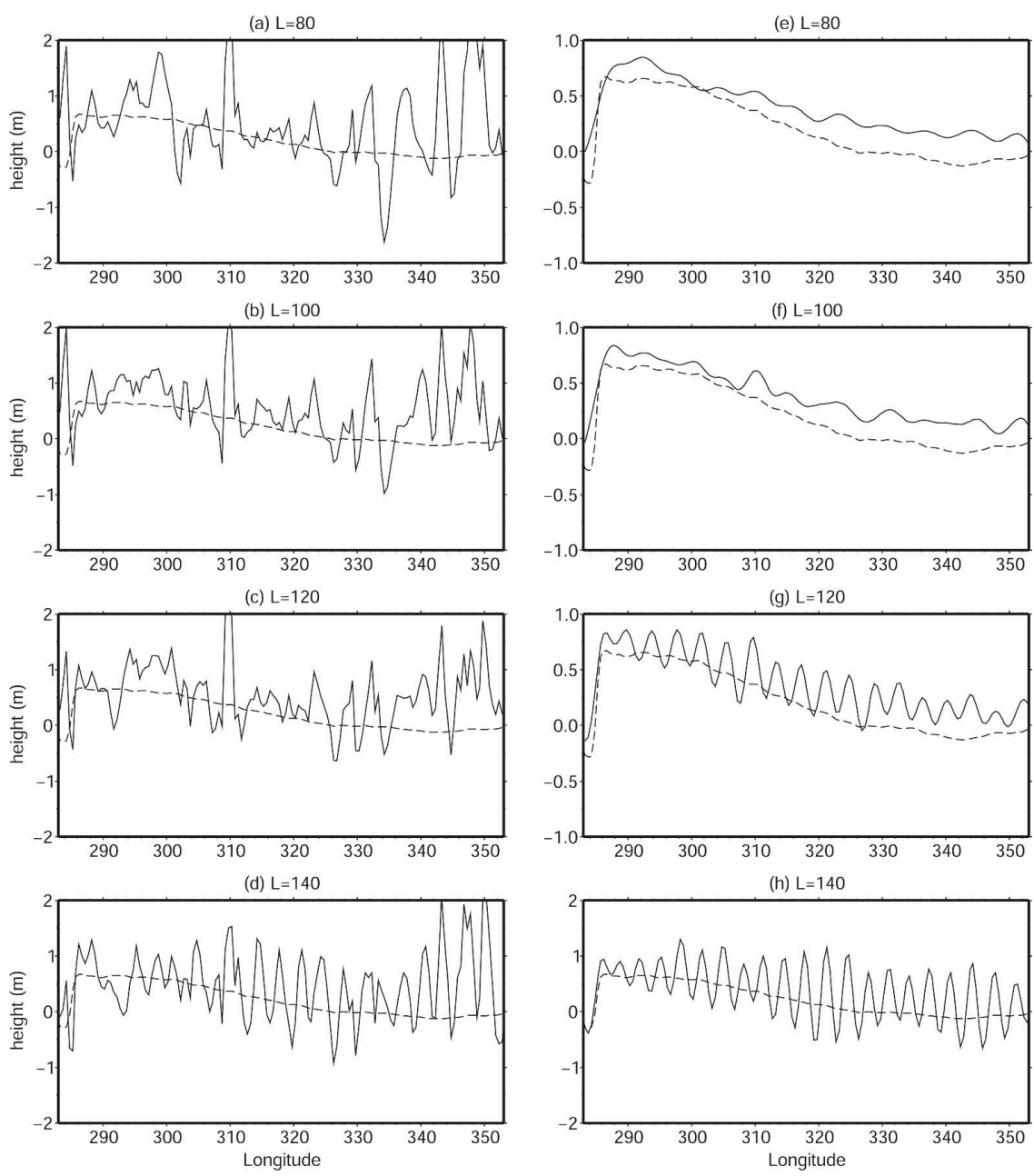

FIG. 5. Zonal sections across the Atlantic at $30^{\circ} \mathrm{N}$ from unfiltered pointwise (left) MDTs and (right) spectral MDTs, with the truncation degree $L$ increasing from top to bottom. For reference, an MDT section from an assimilation experiment using the OCCAM ocean model is also shown (broken line).

would be futile to attempt to obtain surface currents by computing slopes from these profiles. In contrast, for $L=80$ the spectral MDT captures the main gyre features and their magnitudes. The position and slope of the MDT associated with the mean Gulf Stream is now clearly resolved, as is the gentler slope of the interior southward return flow. The gradient of the Gulf Stream is somewhat less than in OCCAM. This is a manifestation of MDT, as opposed to geoid, omission error, and is reduced, just as the geoid omission error is, by increasing $L$. Even with the spectral method, for $L=80$ there are undulations in the downward slope that appear unphysical. These are due to the geoid commission error, a component of the MDT error budget that, in contrast to the omission error component, grows with increasing $L$. The result is that as $L$ is increased beyond degree 80 the spectral MDT rapidly decreases in quality.

An assessment of the relative impact of the geoid omission and commission errors on the MDT calculation can be made by computing the global RMS difference between the pointwise and the OCCAM MDTs for all possible values of $L$ (solid line in Fig. 6a). This, of course, does not quantify the error exactly, only the departure from the OCCAM MDT. However, the close correspondence with CLS01 omission error curve (broken line), which is the RMS difference between the full MSS and the spectral model of the surface truncated at degree $L$ and that serves as a proxy for the geoid omission error, shows that for values of $L$ up to 130 the geoid omission error has the greatest impact on the MDT. Beyond this, the improvement in the quality of 
(a)

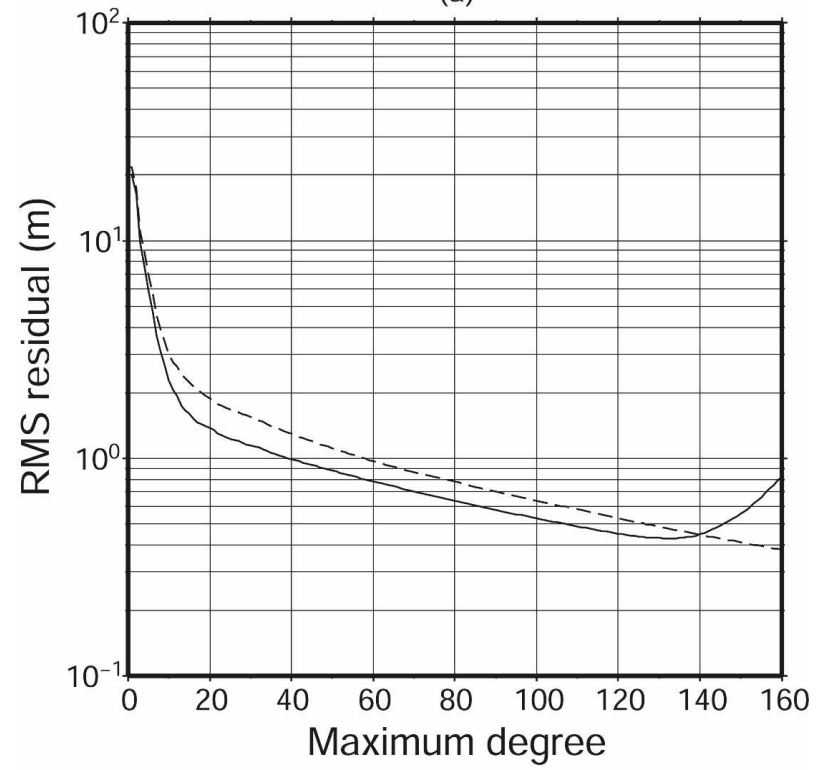

(b)

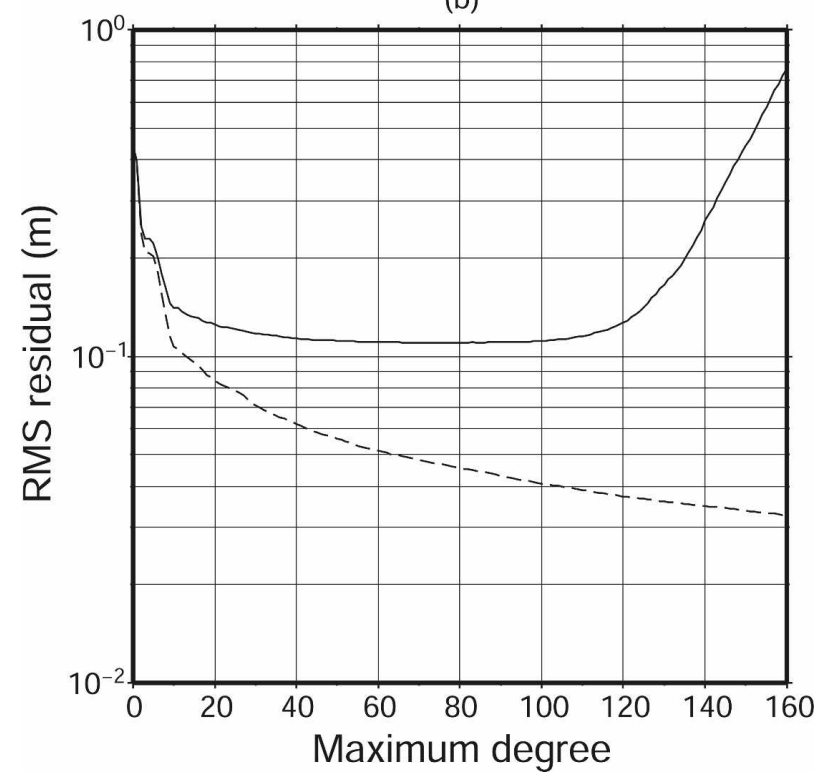

FIG. 6. (a) The RMS difference between the OCCAM MDT and the pointwise MDT as a function of truncation degree $L$ (solid line), and the RMS omission error of the CLS01 MSS as a function of $L$ (broken line). (b) The RMS difference between the OCCAM MDT and the spectral MDT as a function of $L$ (solid line), and the RMS omission error of the OCCAM MDT as a function of $L$. Note the change of scale between the two axes.

the MDT from the reduction of the geoid omission error is outweighed by the exponentially growing geoid commission error. A comparison of the pointwise and spectral MDTs for $L=140$ (Figs. 5d,h), where both MDTs have similar errors, confirms this.

Interpretation of the RMS difference between the spectral and OCCAM MDTs (solid line in Fig. 6b) is not as straightforward as it was for the pointwise case, where either the geoid omission or commission errors dominated. Up to degree 10 , the residual is clearly dominated by the MDT omission error, a fact confirmed by the close correspondence with OCCAM MDT omission error curve (broken line), which is the RMS difference between the full OCCAM MDT and the spectral model of the MDT truncated at degree $L$. Beyond this point, increasing $L$ does not increase the correspondence between OCCAM and the spectral MDT. This is in part because of large-scale, and dynamically related, differences between the two fields, due in large part to different time periods to which the two MDTs refer (1993-96 for OCCAM compared with 1993-99 for the geodetic MDT). Because the MDT omission error is smaller (one-two orders of magnitude) than the geoid omission error, for the spectral MDT the impact of the geoid commission error becomes significant for much smaller values of $L$. In Fig. $6 \mathrm{~b}$ the growing impact of the geoid commission error is reflected in the divergence of the two curves beyond $L=80$.

With the spectral method some problems remain due to the fact that the MDT is discontinuous at land-sea boundaries, which leads to some Gibbs oscillations in the gridded MDT, although these are much smaller than with the pointwise method. Also, depending on our choice of $L$, there will be some noise from the geoid commission error. Hence, the spectral method results in an MDT that still requires some additional spatial smoothing. In the remainder of this section, therefore, we will compare filtered versions of both MDTs to determine if indeed the spectral method yields any advantage over the pointwise method.

\section{a. Filtering method}

A wide range of spatial averaging filters are available, each with its own spectral characteristics. The results presented here are for a Gaussian filter defined in the spatial domain by

$$
f(r)=\exp \left(-r^{2} / 2 \sigma^{2}\right) /(\sqrt{2 \pi \sigma}),
$$

and truncated at $r=10 \sigma$. However, the results obtained were not significantly different from those obtained using other spatial averaging filters in common use (including Hamming and Hanning filters, and a Gaussian filter truncated at $r=2 \sigma$ ). Unlike, a so-called ideal filter, the cutoff frequency of a practical filter can only be defined approximately. The spectral characteristics of the Gaussian filter are defined by its half-weight radius $\beta$, where $f(\beta)=f(0) / 2$, which gives $\sigma=\sqrt{\beta^{2} /(2 \ln 2) \text {. }}$ 
(a)

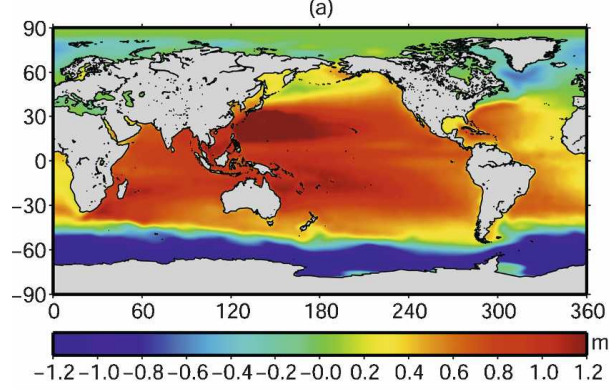

(c)

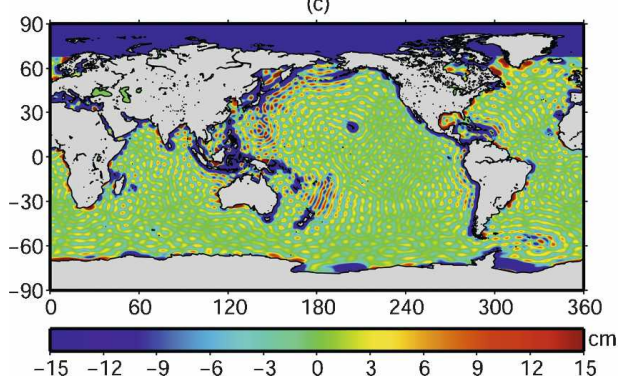

(b)

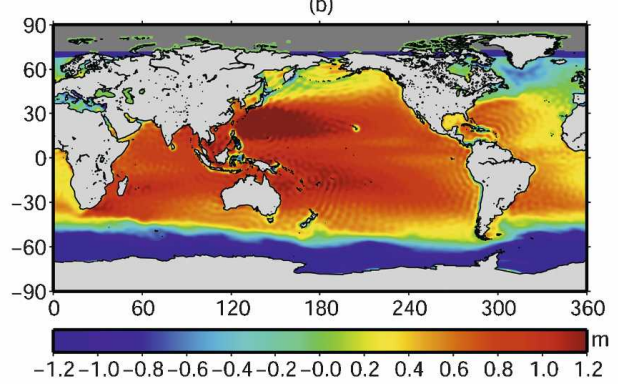

(d)

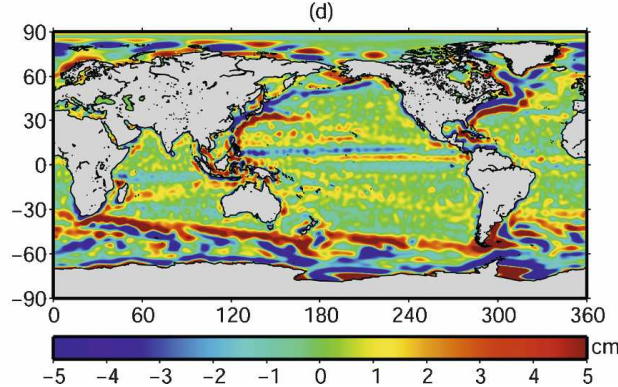

FIG. 7. (a) The spectral MDT expanded to degree 80 and smoothed with a Gaussian filter of 200-km half-weight radius. (b) The pointwise MDT computed with GGM02S expanded to degree 80 and smoothed with a Gaussian filter of 200-km half-weight radius. (c) The difference between the smoothed MDTs shown in (a) and (b). (d) The difference between the spectral MDT shown in (a) and the same MDT, but smoothed with a Gaussian filter of half-weight radius of $300 \mathrm{~km}$.

In addition to the signal attenuation problem mentioned in the introduction, a further filtering issue is how to deal with coastal regions where the filter window overlaps land, because here the MDT is undefined. Setting undefined, land grid points to zero and including these in the filtered value introduces artificial gradients around coasts by smoothing out the step at the land-sea boundary between non-zero values where the MDT is defined and zero values where it is not. In the spatial domain this problem is readily alleviated by excluding undefined land values from the filter. Excluding land values does, however, reduce the averaging area, resulting in undesirable modification of the spectral characteristics of the filter. If the filter radius has been carefully chosen to be no more than is necessary to remove noise over the open ocean, then the latter option may result in ineffective noise suppression in coastal regions. As we shall see, this is a particular problem for the pointwise approach, for which the largest errors tend to occur adjacent to coastlines. Incidentally, the inability to distinguish values over land is one reason why, although possible, we do not filter the MDT in the spectral domain using a spectral filter equivalent to spatial averaging, such as that proposed by Jekeli (1981).

As discussed in the introduction, to avoid degrading the oceanographic signal it is important to find the minimum filter radius that is sufficient to remove noise.
The filter radius $r=r_{0}$ is judged to be sufficient when the difference between an MDT smoothed with a filter of radius $r_{0}$ and with a filter of radius $r=r_{0}+50 \mathrm{~km}$ does not include any patterns characteristic of either the Gibbs oscillations (dominated by decaying oscillations at the boundaries of the MDT) or the commission error (dominated by meridional stripiness). By using the dominant contamination patterns as the criteria by which to find the sufficient filter radius, other noise with spatial scales equal to, or smaller than, the dominant noise components is also effectively suppressed.

\section{b. Comparison of filtered MDTs}

As we have seen, geoid commission errors become a significant error source in the spectral MDT beyond a maximum truncation degree of $L=80$. So here we begin by comparing the spectral and pointwise methods for the $L=80$ case. Using $\beta=20000 / 80=250 \mathrm{~km}$ as a starting point, we found that a half-weight radius of $200 \mathrm{~km}$ was sufficient to effectively suppress the remaining noise in the spectral MDT (see Fig. 7a). The technique of finding the sufficient filter radius is demonstrated by Fig. 8 a. Decreasing the filter radius to 150 $\mathrm{km}$ permits noise of a few centimeters in amplitude, while increasing the radius to $250 \mathrm{~km}$ results in little change to the MDT profile, apart from diminishing the Gulf Stream gradient. This convergence at $200 \mathrm{~km}$ in- 
(a)

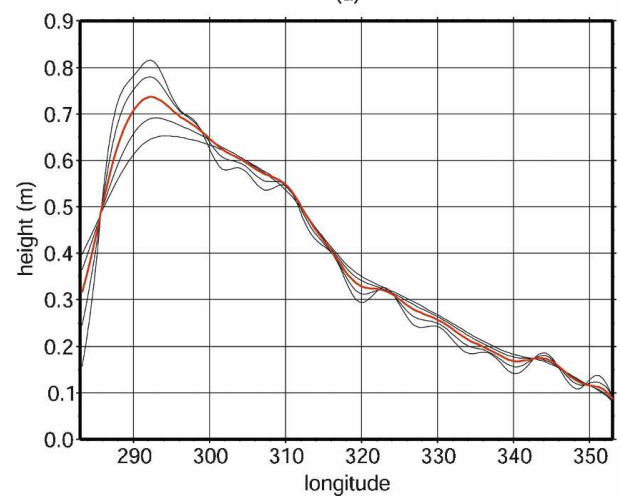

(b)

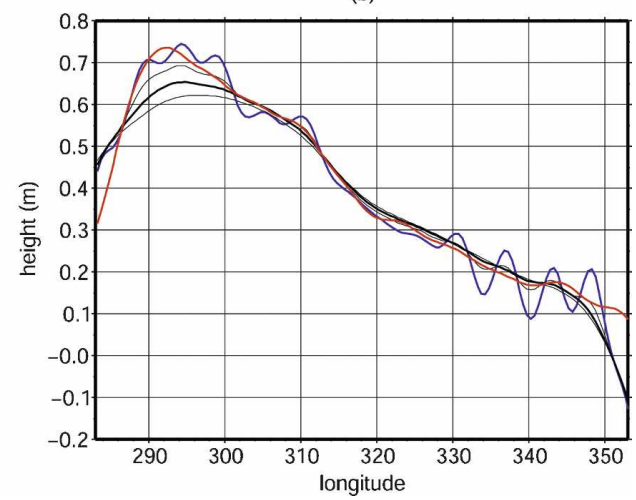

FIG. 8. (a) Zonal sections across the Atlantic at $30^{\circ} \mathrm{N}$ from the spectral $L=80 \mathrm{MDT}$, and Gaussian filtered with half-weight radii ranging from 100 to $300 \mathrm{~km}$ in $50-\mathrm{km}$ steps. The filter radius $(200 \mathrm{~km})$ sufficient to remove the noise is shown in red. (b) Zonal sections across the Atlantic at $30^{\circ} \mathrm{N}$ from the Gaussian filtered pointwise $L=80 \mathrm{MDT}$. The half-weight radius $(300 \mathrm{~km})$ sufficient to remove the noise is shown in bold black, and radii of 250 and $350 \mathrm{~km}$ are shown in black. The half-weight radius $(200 \mathrm{~km})$ sufficient to remove the noise in the spectral MDT is shown in blue. The spectral MDT filtered with a radius of $200 \mathrm{~km}$ is shown in red.

dicates that we have removed the noise, and further filtering only serves to attenuate the ocean signal. Convergence occurs because the dominant noise has a characteristic length scale and amplitude.

Applying a filter of $200-\mathrm{km}$ radius to the $L=80$ pointwise MDT on the other hand, leaves significant contamination remaining (see Fig. $7 \mathrm{~b}$ and also the blue curve in Fig. 8b). From inspection of the MDT and the difference between the MDTs produced by the two approaches (see Fig. 7c) one can clearly see that the residual contamination in the filtered pointwise MDT is due primarily to the nonlocal Gibbs component of the geoid omission error. To remove this noise from the pointwise MDT, applying similar criteria as we did for the spectral MDT, we find $300 \mathrm{~km}$ to be a sufficient filter radius (see Fig. 8b). The fact that the pointwise MDT requires filtering at $300 \mathrm{~km}$ rather than at $200 \mathrm{~km}$, as is required for the spectral MDT, results in an additional attenuation of the gradients corresponding to ocean currents and loss of fine detail. This is demonstrated in Fig. 7d where the $L=80$ spectral MDT filtered at $300 \mathrm{~km}$ has been subtracted from the same MDT filtered at $200 \mathrm{~km}$. The greater filter radius results in a significant additional attenuation of the gradients corresponding to strong currents such as the ACC, the Gulf Stream, and the Kuroshio and its extension. We also see the reduction of currents around the boundaries of the subpolar gyres in both the North Pacific and the North Atlantic, and a further flattening of ridges in the MDT corresponding to the equatorial currents. In places, the attenuation corresponds to a reduction in the MDT gradient of greater than $10 \mathrm{~cm}$ over a distance of a few hundred kilometers. At midlatitudes this cor- responds to an underestimate of geostrophic current speeds of up to $10 \mathrm{~cm} \mathrm{~s}^{-1}$.

The above analysis shows that the growth of geoid commission errors places a limit on the oceanographic scales that can be recovered from the data. Beyond a truncation of $L=80$, the geoid commission errors start to make a significant impact on the spectral MDT, and that a filter radius of $200 \mathrm{~km}$ is required to suppress this noise. (Although the same errors are present in the pointwise MDT at $L=80$, they are insignificant because it is dominated by geoid omission errors.) Now, if we increase the truncation point beyond $L=80$, we decrease the dominant spatial scale of the noise. However, as the summation of Eq. (4) implies, the noise that was present at $L=80$ must remain. Therefore, we still need a filter of at least $200 \mathrm{~km}$ to produce an MDT free from noise (see Fig. 9). For the pointwise method, however, the situation is different because at $L=80$ the pointwise MDT is mainly contaminated by the geoid omission error. This error is reduced by increasing $\mathrm{L}$, and at some value (in the present case $L \approx 140$ ) the geoid commission errors begin to dominate. This will be the same as the geoid commission error affecting the spectral MDT. Therefore, for the reasons that apply to the spectral case, we will need a filter radius of at least $200 \mathrm{~km}$ to remove the geoid commission error. In short, the best we can do, without a more effective method of removing the geoid commission error, is the MDT shown in Fig. 7.

Figure 10 shows that by taking $L=140$ and using a filter radius of $200 \mathrm{~km}$ the difference between the pointwise and spectral approaches becomes insignificant over the open ocean. Yet, as Fig. 10 also shows, prob- 


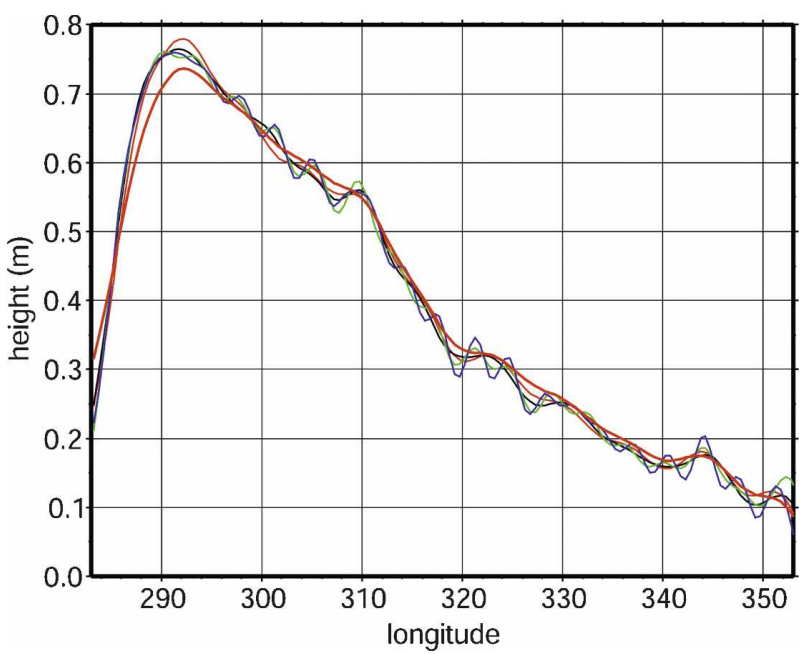

FIG. 9. Zonal sections across the Atlantic at $30^{\circ} \mathrm{N}$ from spectral MDTs $L=80$ (red), $L=100$ (black), $L=120$ (green), $L=140$ (blue), filtered with a Gaussian filter of half-weight radius of 150 $\mathrm{km}$. Shown in bold red is the $L=80$ spectral MDT filtered with the half-weight radius $(200 \mathrm{~km})$ sufficient to remove the noise.

lems with the pointwise approach remain in coastal regions. Problems occur whether values over land are excluded from (top panel) or included in (bottom panel) the averaged values. Interpreted oceanographically, these differences, which are greater than $5 \mathrm{~cm}$ at most coasts, would imply significant coastal currents. When land values are excluded the spectral characteristics of the filter change, such that a filter radius that has been optimized to remove noise over the ocean, is no longer effective. This problem is exacerbated by the fact that, for various reasons, the effect of geoid omission error with the pointwise approach is generally greatest at the coast.

Hawaii, which corresponds to a peak in the geoid, provides a clear example of the problem of how excluding values over land can result in problems with the filtered pointwise MDT. Truncation leads to an underestimation of the gradient of the peak and subsequent oscillations that decay away from the island. The effect of this geoid omission error on the MDT, even for $L=$ 140 , is revealed by subtracting from the unfiltered pointwise MDT the spectral MDT, with $L=140$ in both cases (see Fig. 11a). Around the island the geoid omission error means that the pointwise MDT is underestimated, this is followed by further oscillations of over- and underestimates. With the pointwise approach this additional noise must be removed through spatial filtering. However, the shape of the filter is such that the trough around the island, already greater in magnitude than subsequent oscillations, is amplified still further relative to the subsequent oscillations (see Fig.

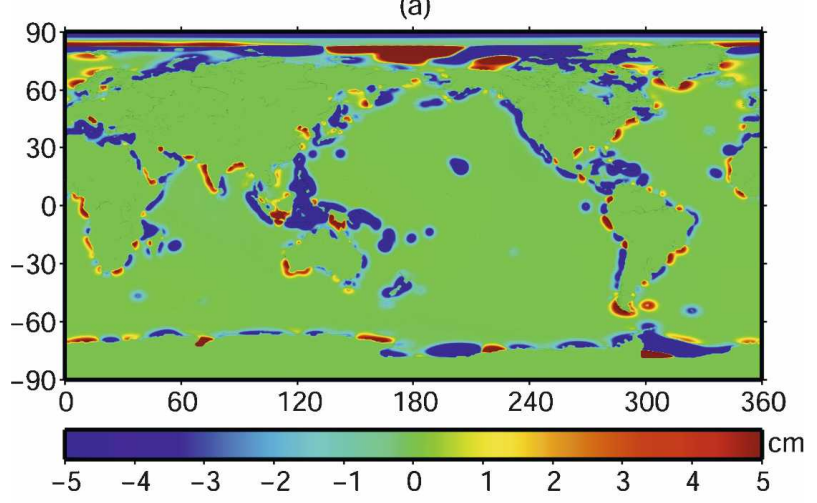

(b)

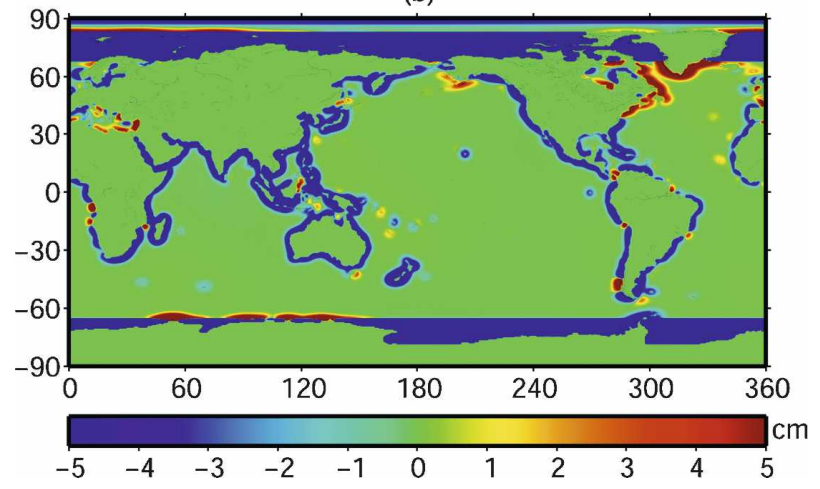

FIG. 10. (a) The residual upon subtracting the $L=140$ spectral MDT from the $L=140$ pointwise MDT, each having been filtered with a Gaussian filter of half-weight radius of $200 \mathrm{~km}$, and not including undefined regions in the filter. (b) As in (a), but for the pointwise MDT including land values in the filter.

11b). This means that the weighted average, which gives the smoothed MDT value, is dominated by the underestimate in the trough. Consequently, while the spectral MDT meets the island (or rather the land mask corresponding to the island) without deviation (black line in Fig. 11d), the filtered pointwise MDT is greatly underestimated (by over $80 \mathrm{~cm}$ ) where it meets the island (red line). Interpreted oceanographically, this implies a strong circulation around the island that, in reality, does not exist. The nature of the omission error here is such that the complement to the omission error trough around the island is a peak over the island, which if included in the weighted average compensates the trough [see Figs. 11c,d (blue curve)]. This greatly reduces the underestimation of the MDT around the island [Fig. 11d (green curve)].

While including land values can reduce problems around small islands such as Hawaii, generally, as Fig. 10 shows, including land values also results in problems. This is because the land values in the pointwise MDT are nothing more than the difference between the EGM96 geoid used as a proxy in CLS01 and the 
(a)

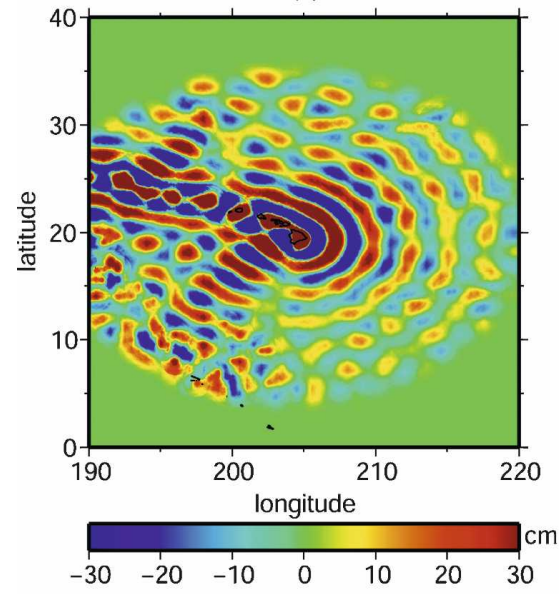

(c)

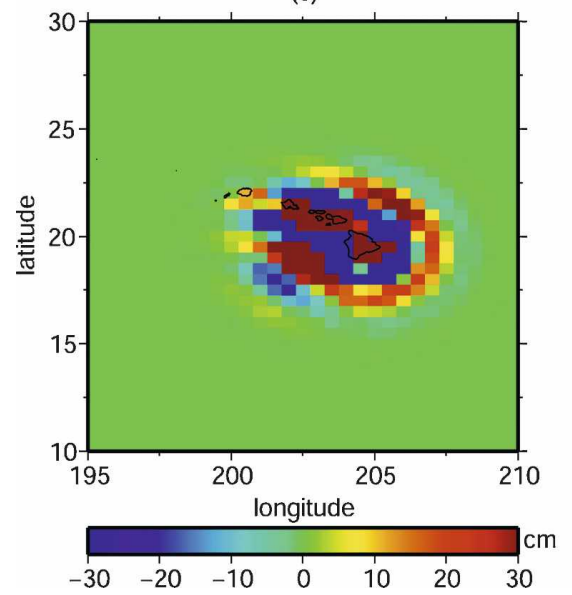

(b)

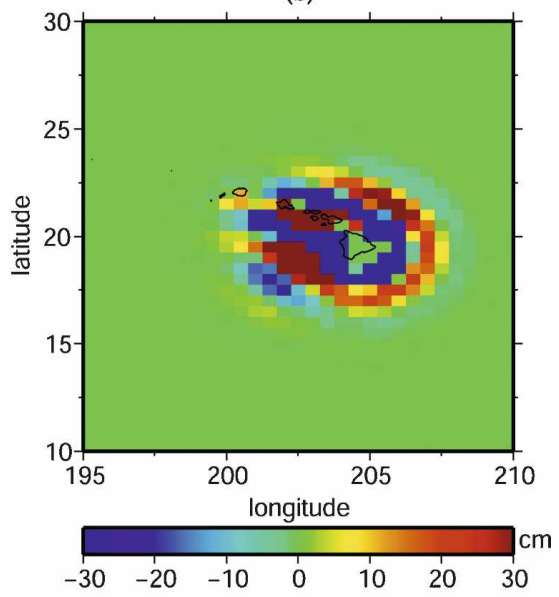

(d)

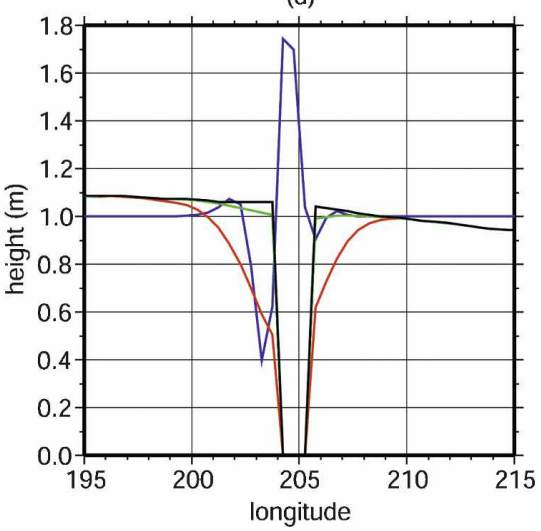

FIG. 11. An examination of the filtering problem around Hawaii. (a) The residual upon subtracting the $L=140$ spectral MDT from the $L=140$ pointwise MDT as seen in the filter window of half-weight radius of $200 \mathrm{~km}$ centered at a point on the western side of Hawaii; this is equal to geoid omission error for $L=140$. (b) As in (a), but weighted by the filter weights normalized to be one at the center of the filter and land masked. (c) As in (b), but without land masked. (d) Zonal sections intersecting Hawaii taken from the center line of the filter. From the $L=140$ spectral MDT (black) and pointwise MDT (red), each filtered with a Gaussian filter of half-weight radius of $200 \mathrm{~km}$ with land excluded, and land included for the pointwise MDT (green). The zonal section of the residual shown in (c), but multiplied by 10 and offset by $1 \mathrm{~m}$ (blue).

GGM02S geoid. This noise, together with the noise in the MDT, tends to average to zero in the filter window, resulting in similar coastal attenuation of the MDT as would occur if zero values were used over land. The fact that the difference between the pointwise and spectral MDTs is generally of opposite sign to the sign of the MDT confirms this.

\section{Summary and conclusions}

Two algorithms for combining MSS and satellitederived geoid data to determine the ocean's mean dy- namic topography (MDT) have been considered in this paper: a pointwise approach, whereby the gridded geoid height field is subtracted from the gridded MSS and the resulting MDT is then spatially smoothed to remove noise; and a spectral approach, whereby the spherical harmonic coefficients of the geoid are subtracted from an equivalent set of coefficients representing the MSS, from which the gridded MDT is then obtained and spatially smoothed to remove residual noise. The essential difference is that with the latter approach the MSS, or rather a hybrid MSS/geoid surface is truncated, a form of filtering, just as with the geoid. This 
ensures that errors of omission resulting from the truncation of the geoid, which are small in comparison to the geoid but large in comparison to the MDT, are matched, and therefore negated, by similar errors of omission in the MSS. This eliminates the need to spatial filter the noise resulting from geoid omission error.

It is shown that geoid errors of omission can be both local and nonlocal in nature. Local omission errors refer to the absence of small-scale physical features, while nonlocal errors of omission are a Gibbs-type effect due to steep gradients in the gravity field where there is topography of substantial relief. Distinguishing these two aspects of the omission error is important because nonlocal effects mean that even steep gradients in the gravity field over land can lead to contamination of the MDT. This means that care is required to use the best proxy over land in the MSS/geoid hybrid. We find that the best results are achieved by using a geoid proxy that is close to that used in the MDT calculation.

In addition to geoid errors of omission, noise in the MDT also arises from geoid errors of commission. Geoid errors of omission decrease with increasing $L$, the truncation degree, while beyond a certain value of $L$ geoid errors of commission, and the noise in the MDT arising from them, grows exponentially. With the pointwise approach, noise in the MDT from omission errors can be reduced by increasing $L$. However, this comes at the expense of increased noise from the geoid commission errors. By eliminating the problem of geoid omission error, the spectral method allows the choice of $L$ to be determined solely by the growth of geoid commission errors. The spectral model of the MDT can be truncated at the point at which geoid commission errors start to become significant. For the GGM02S gravity model used here this is approximately at $L=80$.

Spatial filtering is problematic because in addition to removing noise it also attenuates the steep gradients in the MDT that correspond to the ocean currents we are primarily interested in. Because the degree of attenuation is proportional to the filter width, we wish to use the minimum filter radius that is sufficient to remove noise from the MDT. With the spectral method we do not need to contend with omission error. Rather, we only have to deal with the realized geoid commission error, the magnitude of which we can control with our choice of $L$, and any residual noise, which comes, primarily, from the incomplete cancellation of the Gibbs oscillation that occur because the MSS/geoid hybrid is not identical to the geoid. With the data used in this study we found that for the spectral MDT with $L=80$ the minimum sufficient half-weight radius of a Gaussian filter was $200 \mathrm{~km}$. This compares with a filter radius of $300 \mathrm{~km}$ for the pointwise method when the geoid is truncated at $L=80$. This more severe filtering results in additional attenuation of MDT gradients that for the strongest currents at midlatitudes is equivalent to as much as $10 \mathrm{~cm} \mathrm{~s}^{-1}$.

For the spectral MDT, increasing $L$ beyond degree 80 , only adds errors with shorter spatial scales to the commission errors already present, so we still need a filter of radius at least $200 \mathrm{~km}$ to obtain a smooth MDT. We found that by using $L=140$ we can reduce the geoid omission error noise in the pointwise MDT to the stage where commission errors are dominant and then a $200-\mathrm{km}$ filter is sufficient to remove the remaining noise over the open ocean. However, near coastlines, where noise due to the geoid omission errors is at its worst, spatial filtering is unable to remove the noise properly and the spectral method produces much better results. In previous studies filter radii of 330 (Jayne 2006) and $500 \mathrm{~km}$ (Tapley et al. 2003) have been used, with the attenuation of currents this entails. So we see that a particular benefit of the spectral approach, with a careful attempt made to find the minimum filter radius, is that it allows us to determine, and thereby obtain, the highest possible resolution that can be obtained from the data.

Another possible approach that the spectral method allows depends on the fact that errors of omission in either the geoid or the MSS that propagate into the MDT are not the same as errors of omission in the spectral MDT. Unlike the geoid errors of omission, which just result in noise in the MDT that requires removing, the MDT omission errors have dynamical significance. For instance, we can ask what is the limit beyond which including more terms in the expansion of the spectral MDT results in an insignificant increase in oceanographic information. This depends both on geographical location and on the mean period over which the MDT is computed. Consideration of the spectral MDT profiles (see Fig. 5) makes clear that in extending the truncation point there is a trade-off between increased resolution of the sharp MDT gradients corresponding to strong currents and increased contamination due to the geoid commission errors in regions of broader flows where there are larger-scale but smalleramplitude gradients. This is related to the signal to noise ratio of the coefficients of the geoid model; only in regions where there are actual high-amplitude, shortwavelength features present in the MDT does the signal dominate over noise introduced by including the highorder terms in the expansion of the geoid, while in other regions the errors in the higher-order terms may more than offset improvements gained by their inclusion. With the spectral method we can see therefore that there may be a case for using a regionally variable 
$L$ based on an a priori estimate of the steepness of the MDT gradients. These issues are the focus of ongoing research by the authors.

A further important benefit of the spectral approach is that it eliminates MDT errors due to the geoid omission errors, thereby allowing us to isolate and deal with commission errors separately. In this paper we have dealt with these by limiting the degree and order to which we truncated the expansion of the spectral model of the MDT and by spatial smoothing the gridded MDT. To filter the MDT in a more rigorous fashion would require a strategy based on commission error covariances for both the geoid and the MSS. Presently, such information is not available for either the MSS or the GRACE gravity model. However, it is planned that error covariances will be made available as part of the data release from the upcoming GOCE mission, and how these can best be used to constrain the MDT solution is the subject of ongoing research. (It is worth noting that with the spectral method, unlike the pointwise method, we do not need error covariance information for the geoid omission error.) Ultimately, error covariance information should also allow us to provide a formal error estimate for the MDT, which at present we cannot. Such an estimate is of course important if the MDT is to be optimally combined with other data sources, or assimilated into an ocean model.

Acknowledgments. This work was funded by the U.K. Natural Environment Research Council as part of the research programme "Geodetic oceanography, polar oceanography and sea level," part of Oceans 2025, and is a contribution to the European GRACE Science Working Team. This work was also supported by the European Space Agency GOCE User Toolbox, GUTS project, and we thank the partners of that project for many useful discussions.

\section{REFERENCES}

Emery, W. J., and R. E. Thomson, 2001: Data Analysis Methods in Physical Oceanography. Elsevier, 638 pp.

Fox, A. D., and K. Haines, 2003: Interpretation of water transformations diagnosed from data assimilation. J. Phys. Oceanogr., 33, 485-498.

Hernandez, F., and P. Schaeffer, 2001: The CLS01 mean sea surface: A validation with the GSFC00.1 surface. Tech. Rep., CLS, Ramonville, St Agne, France, 14 pp.

Hoskins, B. J., 1980: Representation of the Earth topography using spherical harmonics. Mon. Wea. Rev., 108, 111-115.
Hughes, C. W., and R. J. Bingham, 2008: An oceanographer's guide to GOCE and the geoid. Ocean Sci., 4, 15-29.

Jayne, S. R., 2006: Circulation of the North Atlantic Ocean from altimetry and the Gravity Recovery and Climate Experiment geoid. J. Geophys. Res., 111, C03005, doi:10.1029/ 2005JC003128.

Jekeli, C., 1981: Alternative methods to smooth the Earth's gravity field. Reports of the Department of Geodetic Science and Surveying 327, The Ohio State University, Columbus, OH, 48 pp.

LeGrand, P., 2001: Impact of the Gravity Field and Steady-State Ocean Circulation Explorer (GOCE) mission on ocean circulation estimates: Volume fluxes in a climatological inverse model of the Atlantic. J. Geophys. Res., 106 (C9), $19597-$ 19610.

Lindberg, C., and A. J. Broccoli, 1996: Representation of topography in spectral climate models and its effect on simulated precipitation. J. Climate, 9, 2641-2659.

Losch, M., and J. Schröter, 2004: Estimating the circulation from hydrography and satellite altimetry in the Southern Ocean: Limitations imposed by the current geoid models. Deep-Sea Res. I, 51, 1131-1143.

Maximenko, N. A., and P. P. Niiler, 2005: Hybrid decade-mean global sea level with mesoscale resolution. Recent Advances in Marine Science and Technology, N. Saxena, Ed., PACON International, 55-59.

Moritz, H., 1980: Advanced Physical Geodesy. Abacus P, 500 pp.

Rio, M. H., and F. Hernandez, 2004: A mean dynamic topography computed over the world ocean from altimetry, in-situ measurements and a geoid model. J. Geophys. Res., 109, C12032, doi:10.1029/2003JC002226.

Smith, D. A., 1998: There is no such thing as "The" EGM96 geoid: Subtle points on the use of a global geopotential model. Int. Geoid Serv. Bull., 8, 17-28.

Stammer, D., and C. Wunsch, 1994: Preliminary assessment of the accuracy and precision of TOPEX/POSEIDON altimeter data with respect to the large-scale ocean circulation. J. Geophys. Res., 99 (C12), 24 584-24 604.

Tapley, B. D., D. P. Chambers, C. K. Shum, R. J. Eanes, J. C. Ries, and R. H. Stewart, 1994: Accuracy assessment of the large-scale dynamic ocean topography from TOPEX/ POSEIDON altimetry. J. Geophys. Res., 99 (C12), 24 60524618.

,,-- S. Bettadpur, and J. C. Ries, 2003: Large scale ocean circulation from the GRACE GGM01 geoid. Geophys. Res. Lett., 30, 2163, doi:10.1029/2003GL018622.

— , and Coauthors, 2005: GGM02-An improved Earth gravity field model from GRACE. J. Geod., 79, 467-478, doi:10.1007/ s00190-005-0480-z.

Webb, D. J., A. C. Coward, B. A. de Cuevas, and C. S. Gwilliam, 1997: A multiprocessor ocean general circulation model using message passing. J. Atmos. Oceanic Technol., 14, 175-183.

Wunsch, C., and E. M. Gaposchkin, 1980: On using satellite altimetry to determine the general circulation of the oceans with application to geoid improvement. Rev. Geophys., 18, $725-745$. 